\title{
Avaliação do Desenvolvimento Geral de Crianças de um a Cinco Anos de Idade Contaminadas por Chumbo
}

\author{
Olga Maria Piazentin Rolim Rodrigues \\ Luciana Esgalha Carnier \\ Universidade Estadual de São Paulo
}

\begin{abstract}
RESUMO
Este trabalho pretendeu investigar, por meio do Inventário Portage Operacionalizado, o desenvolvimento geral e as áreas em defasagem de 60 crianças de um a cinco anos de idade, com nível de contaminação por chumbo no sangue superior a $10 \mu \mathrm{g} / \mathrm{dl}$. Foram avaliadas 15 de cada faixa etária: 1 a 2,2 a 3, 3 a 4 e 4 a 5 anos com índices de contaminação por chumbo até $45,40 \mu \mathrm{g} / \mathrm{dl}$. Os resultados obtidos mostraram que em desenvolvimento geral não se observou defasagens importantes. A análise das áreas específicas apontou que as crianças, independente da faixa etária, apresentaram defasagens importantes em Cognição e Linguagem. Em Cognição, considerando o nível de chumbo no sangue, o resultado foi estatisticamente significante para a faixa de dois a três anos. Os dados mostraram que o desempenho em Cognição piora enquanto que o de Linguagem melhora com a idade. Em Linguagem observaram-se mais crianças em defasagem no nível mais alto de contaminação por chumbo do que em Cognição. O número reduzido de crianças e a ausência dos dados do grupo de controle impedem a afirmação de que os resultados observados, ainda que coerentes com a literatura, devem-se somente aos efeitos da contaminação por chumbo.
\end{abstract}

Palavras-chave: contaminação por chumbo; avaliação de desenvolvimento; Inventário Portage Operacionalizado.

\begin{abstract}
General Development Assessment of one to Five Year Old Children Contaminated by Lead

This study investigated, through the "Inventário Portage Operacionalizado", the general development and the areas in imbalance of 60 children of one to five years, with levels of lead contamination in the blood above $10 \mu \mathrm{g} / \mathrm{dl}$. Of the children in the study, 15 had been evaluated every year: 1 to 2, 2 to 3, 3 to 4 and 4 to 5 years with indices of contamination for lead up until $45,40 \mu \mathrm{g} / \mathrm{dl}$. The obtained results showed that general development did not experience important imbalances. The analysis of the specific areas pointed that the children, independent of age, had presented important imbalances in Cognition and Language. The data had shown that the performance in Cognition worsens while Language improves with age. In Language, one observed more children in imbalance with the highest levels of lead contamination compared to that in Cognition. The reduced number of children and the absence of data from a control group made it difficult to confirm the observed results concerning general development following lead contamination, though it is consistent with the research literature.
\end{abstract}

Keywords: contamination for lead; developmental assessment; Inventário Portage Operacionalizado.

Esta pesquisa é parte do projeto “Avaliação do desenvolvimento geral de crianças de um a cinco anos de idade contaminadas por chumbo", desenvolvido por um grupo de professores e alunos do Curso de Psicologia da UNESP-Bauru, desde 2003.

O chumbo é um metal pesado que tem sido amplamente utilizado pelo homem e, atualmente, é usado na fabricação de baterias, aditivos em gasolina, munição, tintas, soldas etc. A exposição ocupacional tem sido intensivamente estudada uma vez que intoxicações graves podem resultar em seqüelas importantes para trabalhadores que têm contato direto ou indireto com o metal (Cordeiro \& Lima-Filho, 1995). Todavia, a exposição ambiental também pode levar a intoxicações graves, se cuidados especiais não forem tomados (Quitério e cols., 2003). A contaminação por chumbo, chamada comumente de saturnismo, acontece porque o chumbo, material estranho ao organismo humano, não é eliminado espontaneamente, acumulando-se nos 
ossos, no sangue e no sistema nervoso. Malta, Trigo e Cunha (2000) referem que, ainda que tratamento e ausência de chumbo no ambiente sejam providenciados, a eliminação deste metal é extremamente lenta, demorando até 10 anos para se efetivar.

Estudos indicam que os efeitos do chumbo são os mesmos tanto para a população em geral quanto para a população exposta ocupacionalmente. Porém, é importante fazer a distinção entre adultos e crianças, uma vez que a suscetibilidade ao metal é diferente entre esses dois grupos. Moreira e Moreira (2004) colocam que a exposição pode começar ainda no útero, caso a mãe tenha chumbo em seu organismo, uma vez que o metal atravessa a barreira placentária, e aumentar após o nascimento, através de inúmeras fontes. Segundo esses autores, nas crianças, os efeitos críticos atingem o sistema nervoso, enquanto nos adultos, com exposição ocupacional excessiva ou mesmo acidental, tais efeitos se fazem presentes na neuropatia periférica e na nefropatia crônica.

Crianças são especialmente vulneráveis aos efeitos do chumbo. Bellinger (1995) afirma em seus estudos que, mesmo quantidades relativamente pequenas de chumbo, podem causar rebaixamento permanente da inteligência em crianças, resultando em prejuízos acadêmicos e distúrbios psicológicos, uma vez que ao agir sobre o sistema nervoso, o metal pode bloquear ou dificultar a transmissão nervosa, podendo haver perda de memória e dificuldade de concentração. A sensibilidade das crianças aos efeitos da exposição se dá, segundo Yule e Lansdown (1993) por que: a) consumo por quilo de peso é maior do que nos adultos; b) crianças colocam objetos que estão no chão, na boca, com maior freqüência; c) a absorção do chumbo pelo organismo da criança é maior do que do adulto; d) crianças pequenas estão em desenvolvimento rápido e constante, tornando-as mais vulneráveis aos efeitos do chumbo. Gutiérrez (2001) concorda com os dados acima quando coloca que a intoxicação nas crianças se dá pela ingestão, a partir das suas mãos sujas com pó, alimentos, objetos etc. contaminados. Thacker, Hoffman, Smith, Steinberg e Zack (1992) lembram que, em geral, o público mais afetado, vive, também em áreas mais pobres, estando exposto além do chumbo a condições menos favorecidas de vida. Ainda que alguns acidentes, resultando em contaminação por chumbo de populações infantis, tenham acontecido no Brasil, não temos resultados publicados que apontem para os efeitos desta contaminação no desenvolvimento das crianças (Silvany, 1996; Campanili, 2001).
O meio ambiente tem um papel importante no desenvolvimento da criança acelerando-o ou retardandoo. O comportamento é determinado por uma série de fatores, entre eles fatores internos, como a maturação e a hereditariedade, e externos, como o ambiente que o cerca, e seus diversos componentes, e suas experiências com este. Uma avaliação do desenvolvimento infantil, para ser adequada, deve levar em conta as condições ambientais e de oportunidade a que estão expostas as crianças (Bee, 1986, 1997). A contaminação do chumbo pode ser uma variável ambiental prejudicial ao desenvolvimento adequado da criança, uma vez que atua, de modo negativo, em enzimas, hormônios etc.

A época da contaminação também gera controvérsias quanto à gravidade de seus efeitos. Moreira e Moreira (2004) colocam que, contaminações prénatais estariam influenciando no nascimento prematuro dos bebês, além de parecerem confirmar um baixo peso dos recém nascidos. Wasserman (1995) aponta, em seu texto, que a exposição pós-natal está mais fortemente relacionada a perdas cognitivas que a exposição pré-natal. Shaheen (1984, citado por Wasserman, 1995), apresentou dados sugerindo que crianças que estiveram expostas ao chumbo até dois anos de idade, teriam déficits na área da linguagem, enquanto que, crianças que sofreram contaminação após esta idade, teriam mais dificuldades com relação à integração visomotora.

Tais efeitos podem ser avaliados a partir da utilização de escalas de desenvolvimento infantil, que tem se mostrado útil na medida em que dá parâmetros para comparação do desenvolvimento da criança com os pares da mesma idade cronológica. Dentre as várias escalas de desenvolvimento utilizadas para detecção de distúrbios do desenvolvimento infantil, destaca-se o Inventário Portage Operacionalizado (IPO), descrito por Williams e Aiello (2001). Este inventário fornece uma visão global do desenvolvimento da criança dividindo-o em cinco grandes áreas: cognição, desenvolvimento motor, linguagem, socialização e autocuidado, possibilitando a detecção de áreas que devem ser objeto de intervenção. Prevê, inclusive, avaliações seqüenciais, como forma de monitorar o desenvolvimento da criança a partir da introdução de procedimentos de intervenção. É um inventário relativamente fácil de ser aplicado considerando que as instruções foram definidas operacionalmente e os critérios explicitados cuidadosamente. Além disso, o material se destinava, inicialmente, para o uso por pais de crianças com atraso no desenvolvimento. 
Almeida (2003) e Rodrigues, Almeida e Ribeiro (2003) conduziram estudos para avaliar o desenvolvimento de crianças de um a três anos de idade contaminadas por chumbo utilizando o Inventário Portage Operacionalizado e observaram que as crianças apresentaram desempenho abaixo do esperado em Linguagem e Cognição. As crianças de dois a três anos tinham desempenho, nestas áreas, piores do que as crianças de um a dois anos sugerindo que a defasagem pode aumentar com a idade, para crianças contaminadas por chumbo.

Figueiredo, Ribeiro, Rodrigues, Ribeiro e Almeida (2003) desenvolveram um estudo que teve como objetivo investigar a ocorrência de diferenças no desenvolvimento infantil de 48 crianças, de um a seis anos de idade, considerando o nível de contaminação por chumbo. Ainda que preliminares, os resultados obtidos parecem indicar que, para ambos os grupos, na medida em que a idade avança há uma maior defasagem entre o resultado obtido e o esperado indicando os efeitos da contaminação sobre o desenvolvimento infantil, principalmente nas áreas de linguagem e cognição, tanto considerando o nível de chumbo quanto à idade dos participantes.

Alves, Rodrigues, Troijo, Kusumi e Ribeiro (2004) analisaram o efeito do nível do chumbo no desenvolvimento cognitivo de 123 crianças, com nível acima de $10 \mu \mathrm{g} / \mathrm{dl}$, de ambos os sexos, com idade variando de sete a 73 meses, utilizando os resultados do Inventário Portage Operacionalizado (IPO). Para comparar os efeitos do chumbo estabeleceu-se um ranking das crianças por nível de chumbo, do mais alto para o mais baixo. Identificou-se, então, dois grupos de crianças. No Grupo 1 estavam as 30 crianças com nível mais alto de chumbo e no Grupo 2, as 30 crianças com nível mais baixo. Em ambos os grupos, as idades variaram de 10 a 71 meses, com 13 meninas e 17 meninos em cada um deles. Encontrou-se, então, a diferença, para mais ou para menos, para cada um, a partir do número de pontos esperados para sua idade. Os resultados obtidos mostraram que para o Grupo 1, com nível mais alto, a diferença entre o esperado e o obtido foi de $-0,63$ pontos enquanto que, para as crianças com nível mais baixo foi de +9 pontos. No Grupo 1 , $60 \%$ dos sujeitos apresentaram desempenho abaixo do esperado para sua idade, enquanto que no Grupo $2,37 \%$ apresentaram esta condição. Os resultados parecem indicar o efeito do chumbo sobre o desempenho cognitivo das crianças avaliadas.

Alves, Rodrigues, Figueiredo, Kusumi e Ribeiro (2004) avaliaram o desenvolvimento cognitivo de crianças com nível de chumbo no sangue superior a $10 \mu \mathrm{g} / \mathrm{dl}$, de ambos os sexos, com idade variando de um a seis anos, utilizando o Inventário Portage Operacionalizado (IPO). Os sujeitos foram divididos em grupos por idade: Grupo 1, até dois anos; Grupo 2, de dois a três anos; Grupo 3, de três a quatro anos; Grupo 4, de quatro a cinco anos e Grupo 5, de cinco a seis anos. Considerando os resultados obtidos, observou-se que no Grupo 1,56\% das crianças; no Grupo 2,41\% das crianças; no Grupo 3,59\% das crianças; no Grupo 4,78\% das crianças e, no Grupo 5, 88\% das crianças estavam abaixo do esperado para a sua idade cronológica. Estes dados mostram que à medida que as crianças crescem mais seu desempenho se distancia do esperado para sua idade cronológica. Os resultados obtidos parecem indicar que, para esta população contaminada por chumbo, quanto maior a idade, maior o efeito da contaminação. Porém, considerando que outras variáveis como pobreza, falta de escola, estão presentes para esta população, há que se conduzir estudos com grupos-controle para confirmar ou não se os resultados obtidos se devem à contaminação por chumbo.

O presente projeto pretende ampliar os resultados dos estudos acima relatados avaliando o desenvolvimento geral de crianças de um a cinco anos. São objetivos específicos deste projeto: 1) identificar as áreas de desenvolvimento (motor, autocuidado, cognição, linguagem e socialização) em defasagem; 2) comparar o índice de defasagem de cada uma das áreas com o nível de chumbo detectado no sangue e, 3) comparar o desenvolvimento das crianças em todas as áreas por faixa etária.

\section{MÉTODO}

\section{Participantes}

Participaram do projeto 60 crianças na faixa de um a cinco anos de idade, sendo 15 de cada faixa etária: um a dois anos; dois a três anos, três a quatro anos e quatro a cinco anos; com exposição ambiental ao chumbo, selecionadas a partir da demanda já identificada de 316 crianças de zero a 12 anos. Estas crianças estão sendo encaminhadas ao um centro de Psicologia, de uma universidade pública, pela Diretoria Regional da Saúde (DIR X), de uma cidade de médio porte do interior do Estado de São Paulo, desde maio de 2002, com nível de chumbo no sangue acima de $10 \mu \mathrm{g} / \mathrm{dl}$. A escolha desta faixa etária se deve ao fato de que há poucos estudos em crianças pré-escolares com altos 
níveis de chumbo além dessa fase estar relacionada a marcos importantes no desenvolvimento, tais como, aquisição e desenvolvimento da marcha e linguagem e o desenvolvimento de habilidades básicas para a aprendizagem das atividades acadêmicas.

\section{Materiais e Procedimento}

Os dados foram coletados em salas para atendimento infantil, no referido Centro de Psicologia. Para a coleta de dados iniciais foram utilizados: Roteiro de anamnese simplificada, contendo informações como: dados familiares, nível socioeconômico, há quanto tempo reside no local, desenvolvimento pré-natal, pós-natal e atual da criança, escolaridade, relacionamento familiar etc.; Termo de Consentimento Livre e Esclarecido, autorizando a utilização dos dados coletados na avaliação da criança na pesquisa, esclarecendo questões quanto à participação voluntária, explicitando questões éticas quanto ao anonimato dos participantes; Inventário Portage Operacionalizado (Williams \& Aiello, 2001), composto por folhas de registro das áreas de desenvolvimento avaliadas possuindo, ao todo, 580 comportamentos a serem observados na criança, sendo estes comportamentos subdivididos em seis grandes áreas que são: a) estimulação infantil; b) desenvolvimento motor; c) autocuidados; d) cognição; e) socialização e, f) linguagem. Para a aplicação do Inventário Operacionalizado Portage foram utilizados materiais diversos (jogos, bonecas, bolas, bichos de plástico, brinquedos de casinha, carrinhos, lápis de cor, livros de estórias etc.), sugeridos pelo protocolo de avaliação, propostos para as idades: um a dois anos, dois a três anos, três a quatro anos e cinco a seis anos de idade. Fazem parte, também, deste material, protocolos, por área, para registro das respostas da criança.

Com base em listagem de crianças contaminadas, fornecida pelo órgão público responsável, foi definida a amostra a ser estudada. Os responsáveis pelas crianças identificadas, foram contatados e convidados a participar do projeto. Após a compreensão e aceite, o responsável assinou o Termo de Consentimento Livre e Esclarecido.
Os pais responderam a anamnese e, em seguida, a criança juntamente com seus pais (ou pelo menos um deles) foi conduzida a uma sala de atendimento infantil. Inicialmente o aplicador promoveu diálogos e ações facilitadoras para a interação positiva e de confiança com a criança que foi avaliada. Na medida em que uma relação empática se estabelecia entre o aplicador e a criança era iniciada a aplicação do Inventário Portage Operacionalizado, de acordo com as orientações explicitadas no manual.

\section{Resultados e Discussão}

A avaliação do desenvolvimento geral de crianças de um a cinco anos contaminadas por chumbo pretendia: a) identificar as áreas de desenvolvimento (motor, autocuidado, cognição, linguagem e socialização) em defasagem; b) comparar o índice de defasagem de cada uma das áreas com o nível de chumbo detectado no sangue; c) comparar o desenvolvimento das crianças em todas as áreas por faixa etária.

Nesta primeira etapa da análise descreveremos o desempenho geral e em cada área avaliada (Cognição, Linguagem, Desenvolvimento Motor, Socialização e Auto Cuidado), das crianças de um a dois anos, de dois a três anos, de três a quatro anos e de quatro a cinco anos no IPO, considerando o desempenho obtido comparado ao desempenho esperado. As crianças estão ordenadas por idade, da mais nova para a mais velha, em cada faixa etária. Para esta análise considerou-se acima do esperado quando o desempenho estava $10 \%$ acima do total de pontos para a idade, dentro do esperado, quando o desempenho estava $9 \%$, tanto abaixo quanto acima e, abaixo do esperado, quando o desempenho estava $10 \%$ abaixo.

Analisando o desempenho geral, como mostra a Figura 1, observa-se que $53 \%$ das crianças de um a dois anos de idade estavam acima do esperado; de dois a três anos, $53 \%$ encontravam-se dentro do esperado; de três a quatro anos 60 \% dentro do esperado e de quatro a cinco anos 80 \% estavam dentro do esperado. 


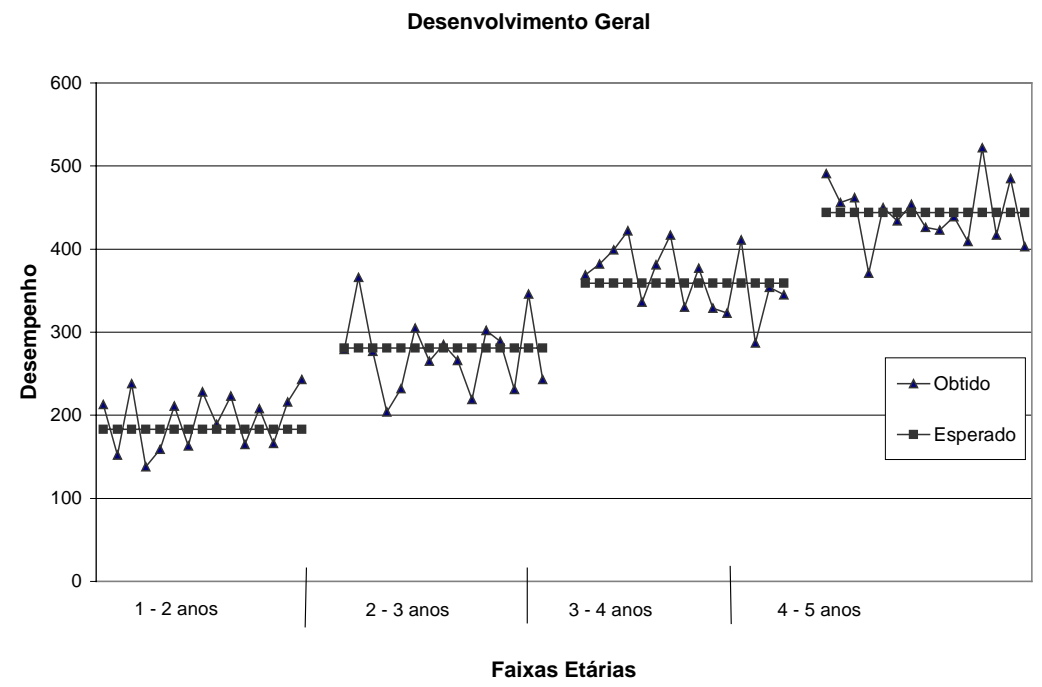

Figura 1. Desenvolvimento geral das crianças de todas as faixas etárias.

A análise do desenvolvimento geral dos quatro grupos de idade mostra que o grupo de crianças de dois a três anos apresentou o pior desempenho. No geral, a maioria das crianças das idades de um a dois, de três a quatro e de quatro a cinco anos ficou dentro ou acima do esperado. Porém, análises específicas das áreas avaliadas podem mostrar se esta tendência continua presente nas mesmas ou se existem áreas em que o desempenho se encontra muito acima e outras muito abaixo do esperado e, que em desenvolvimento geral, o resultado se apresenta dentro da média.

Dentro do mesmo critério utilizado para a análise do desenvolvimento geral, será descrito o desempenho das crianças em cada uma das áreas específicas de desenvolvimento avaliado.

Considerando o Desenvolvimento Motor, observase que $93 \%$ das crianças de um e dois anos; $87 \%$ das crianças de dois a três anos; $100 \%$ das crianças de três a quatro e de quatro a cinco anos de idade apresentaram desempenho acima ou dentro do esperado. Este bom desempenho provavelmente se deve ao fato de as crianças avaliadas morarem em bairros afastados do centro da cidade, nos quais as ruas, em sua grande maioria, são de terra, e apresentam pouco tráfego, dando assim às crianças a liberdade de brincarem fora do espaço restrito de seus lares.

Quanto ao desempenho na Socialização, observase que a maioria das crianças, de todas as faixas etárias, apresentaram um desempenho dentro do esperado, sendo $60 \%$ das de um a dois anos, três a quatro anos e quatro a cinco anos, e 73\% das de dois a três anos.
Na área de Autocuidado, das crianças pertencentes à faixa etária de um a dois anos, a maioria (67\%) apresentou um desempenho acima do esperado; $60 \%$ das de dois a três anos e quatro a cinco anos estavam dentro do desempenho esperado, assim como 53\% das crianças de três a quatro anos de idade. $\mathrm{O}$ alto índice de crianças de 1 a 2 anos acima do esperado pode se dever ao fato de que nesta idade os comportamentos referentes a esta área são obtidos através de informações dadas pelas mães e não a comportamentos observados nas sessões de avaliação.

$\mathrm{Na}$ área de Linguagem, como mostra a Figura 2, nota-se que $47 \%$ das crianças de um a dois anos apresentaram desempenho acima do esperado, enquanto entre as de dois a três anos e de três a quatro anos apenas $27 \%$ das crianças se encontraram nesta faixa de desempenho e nas de quatro a cinco anos apenas $13 \%$ das crianças apresentaram tal desempenho. 6\% das crianças com idade entre um e dois anos e $20 \%$ das entre dois a três anos, tiveram desempenho dentro do esperado, enquanto $60 \%$ e $80 \%$ das crianças de três a quatro e quatro a cinco anos, respectivamente apresentaram desempenho semelhante. Apresentaram desempenho abaixo do esperado, $47 \%$ das crianças de um a dois anos e $53 \%$ das de dois a três anos de idade e apenas $13 \%$ das de três a quatro anos e $7 \%$ das de quatro a cinco anos. Observa-se que, nesta área, os grupos de um a dois anos e de dois a três anos apresentaram defasagens importantes, porém, nota-se que o grupo de crianças de dois a três anos se afastaram mais do desempenho esperado. 


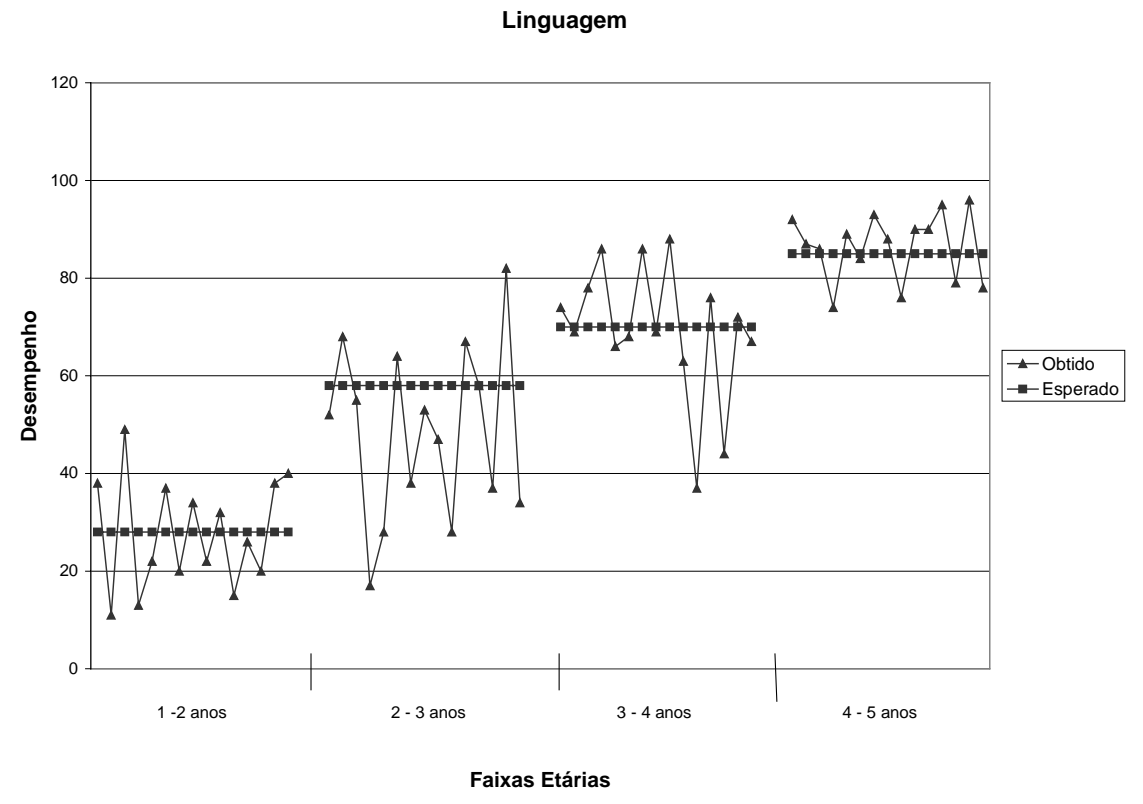

Figura 2. Desempenho em Linguagem.

É importante ressaltar que, para as crianças pequenas, é difícil observar os comportamentos avaliados em Linguagem. As mães, então, são usadas como informantes. A discrepância observada nas idades de 1 a 2 anos e 2 a 3 anos pode ser resultado de informações pouco verídicas dadas pelas informantes ou a não-resposta da criança em situações que poderiam ser observadas, por falta de familiaridade das crianças com o avaliador e com o ambiente, ainda que até cinco sessões ocorreram com sujeitos desta faixa etária.

O desempenho na área da Cognição, como mostra a Figura 3, observa-se que $20 \%$ das crianças apresentaram desempenho acima do esperado, para as faixas etárias de um a dois anos e de dois a três anos, e ape- nas $13 \%$ e $7 \%$, respectivamente, das crianças com idade entre três a quatro anos e quatro a cinco anos apresentaram tal desempenho; 33\% e 40\% das crianças, de um a dois anos e de dois a três anos respectivamente, e $27 \%$ e $20 \%$ das crianças de três a quatro anos e quatro a cinco anos respectivamente, têm desempenho dentro do esperado. Com desempenho abaixo do esperado encontravam-se $47 \%$ das crianças de um a dois anos, $40 \%$ das de dois a três anos, $60 \%$ das de três a quatro anos e $73 \%$ das crianças de quatro a cinco anos. Pode-se dizer que o nível de desempenho abaixo do esperado para a idade é alto para todas as idades, uma vez que ultrapassa os $40 \%$ em todas faixas etárias. 


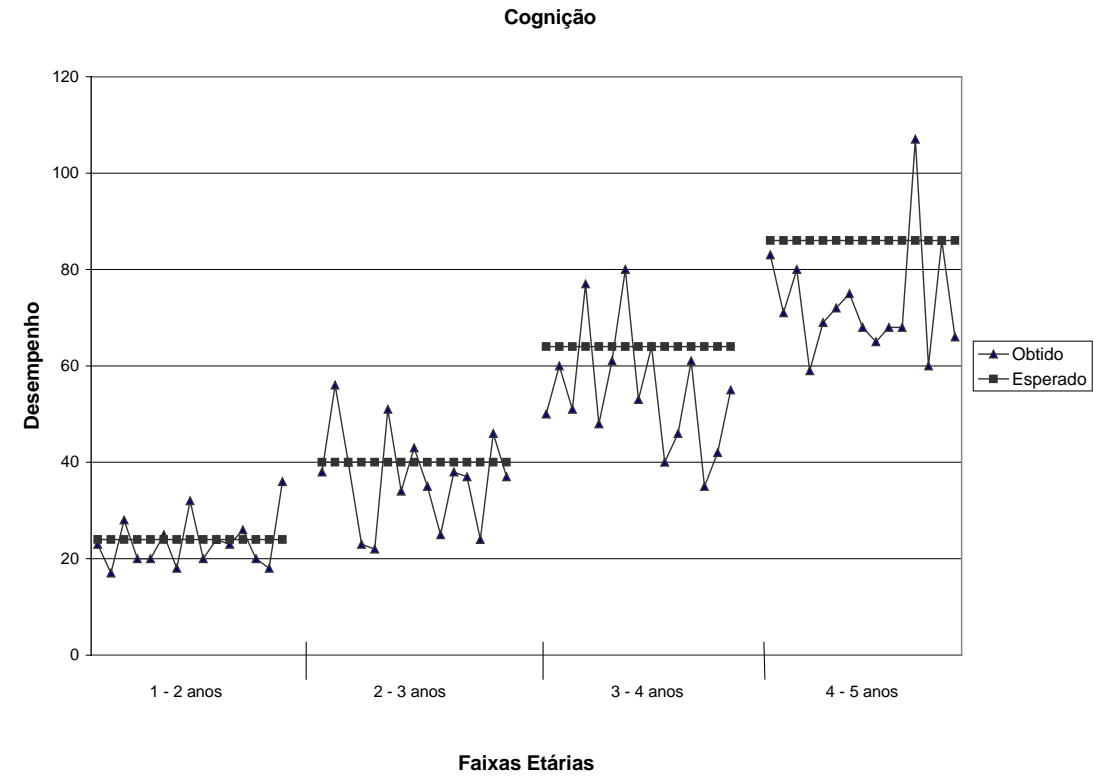

Figura 3. Desempenho em Cognição.

Na Figura 4, nota-se que crianças com idade entre um e dois anos e de dois a três anos de idade apresentaram maior defasagem nas áreas de Linguagem (47\% e $53 \%$ respectivamente) e Cognição ( $47 \%$ e $40 \%$ respectivamente), enquanto as crianças de três a quatro e de quatro a cinco anos de idade apresentaram maior defasagem na área da Cognição (60\% e 73\% respectivamente). Na área dos Autocuidados, a defasagem apresentada ficou entre 20 e 33\%. Nas áreas de Socialização e Desenvolvimento Motor não se observou defasagem para as faixas etárias estudadas.

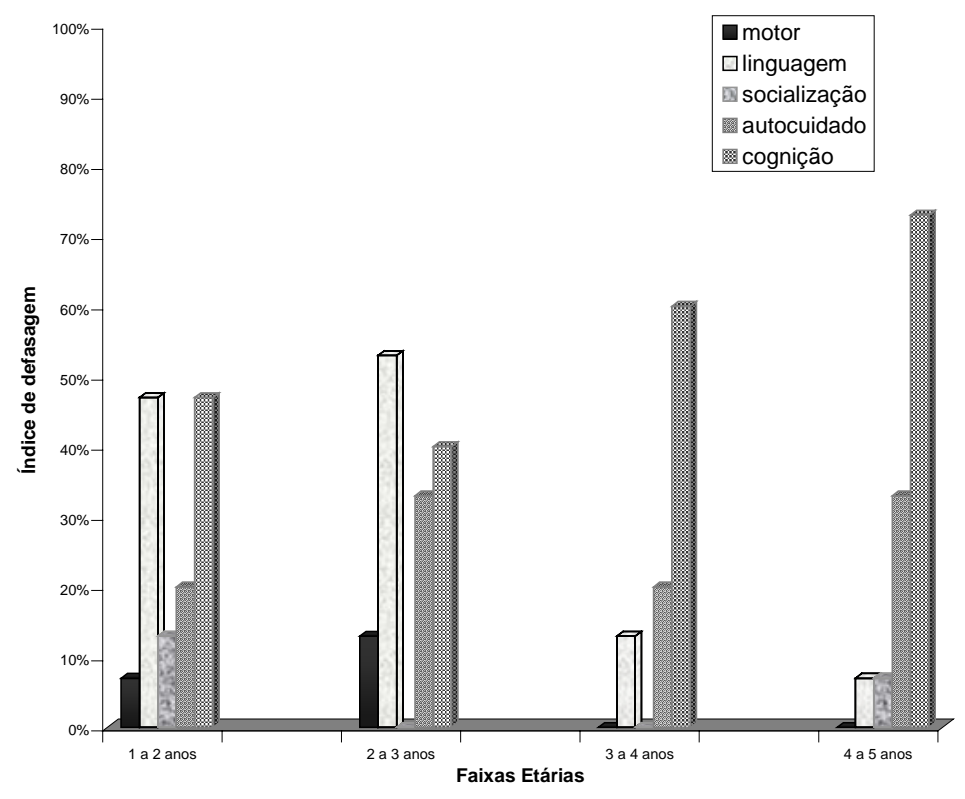

Figura 4. Índice de defasagem em cada área de desenvolvimento. 
Considerando as defasagens por área apresentadas por todos os sujeitos desse estudo, constatou-se que as áreas defasadas são as da Cognição e Linguagem, seguidas, em ordem decrescente, por Autocuidados, Desenvolvimento Motor e Socialização.

Dentro do mesmo critério utilizado acima, analisou-se o desempenho das crianças nas áreas de desenvolvimento que apresentaram maior comprometimento (Linguagem e Cognição) comparado com o nível de chumbo apresentado. Para tal, dividiu-se as crianças em dois grupos, de acordo com o nível de chumbo. O Nível 1 engloba 43 crianças que têm índice de contaminação entre 10 e 19,9 $\mu \mathrm{g} / \mathrm{dl}$ de sangue e o Nível 2 engloba 17 crianças com índices de contaminação superiores a $20 \mu \mathrm{g} / \mathrm{dl}$ de sangue. Em Linguagem observou-se que a porcentagem de crianças com desempenho abaixo da média mais do que dobrou do nível I para o nível II, indicando uma tendência de que a contaminação parece influenciar no desempenho desta área, e, possivelmente, sua concentração também (Figura 5).

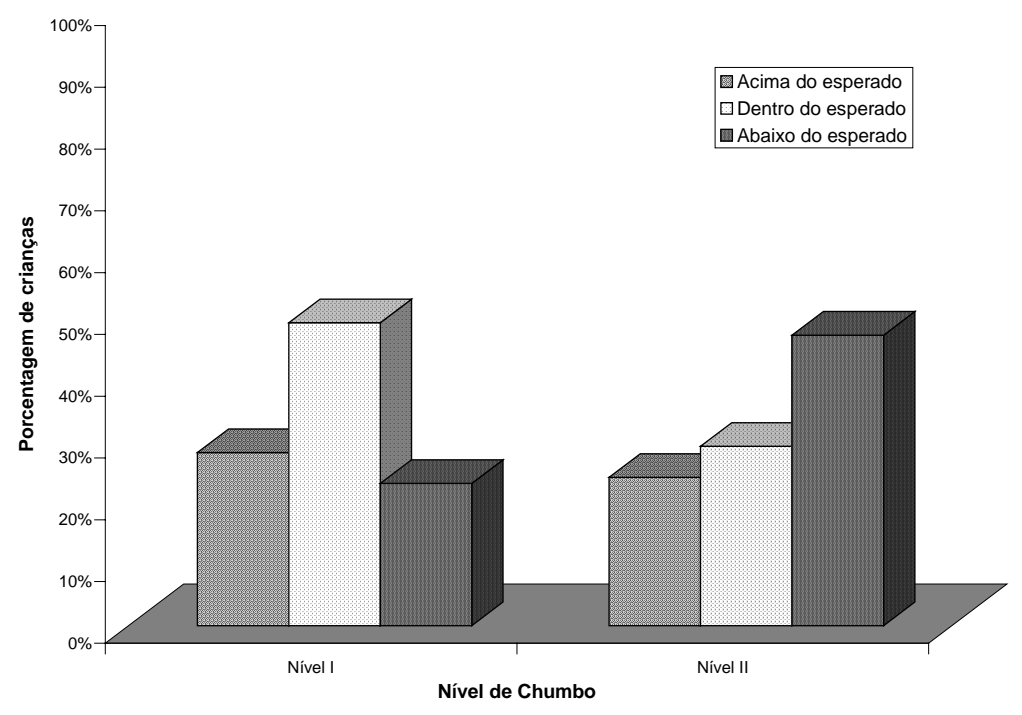

Figura 5. Desempenho em Linguagem em defasagem comparado ao nível de chumbo das crianças.

Para uma análise mais detalhada de tais fatos, a Figura 6, mostra o desempenho na área da Linguagem dividida nas faixas etárias estudadas. Nesta, nota-se que as faixas etárias de um a dois anos e de dois a três anos foram as que apresentaram maior porcentagem de crianças com desempenho abaixo do esperado, o que pode ser novamente explicado por haver dificuldade de observar tais comportamentos e usar as mães como informantes, porém há a corroboração de tal fato com os estudos feitos por Shaheen (1984, citado por Wasserman, 1995), o qual afirma que crianças que estiveram expostas ao chumbo até dois anos de idade, teriam déficits na área da linguagem. Além disso, pode-se observar, comparando-se os grupos de crianças estudados, que as crianças na faixa etária de dois a três anos, são as que apresentaram o mais baixo índice de desempenho na área da linguagem. Esta questão, contudo, pode dever-se a pouca estimulação ambiental que resulta numa linguagem mal elaborada aquém do esperado para essa idade cronológica. Por isso, não há dados suficientes para afirmar que o desempenho obtido, nesta área de desenvolvimento, está relacionado ao nível de chumbo encontrado no sangue. 


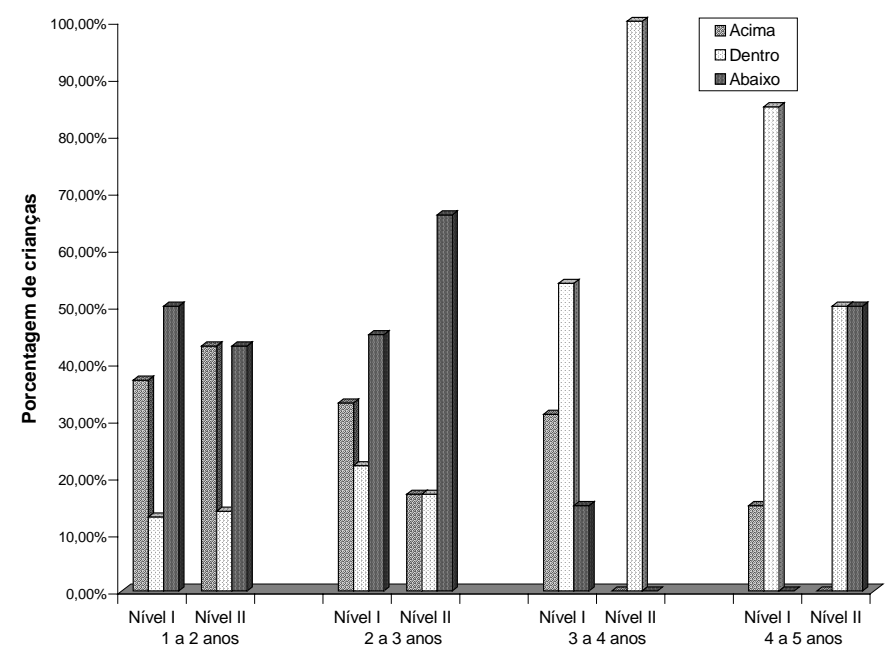

Figura 6. Desempenho em Linguagem e nível de chumbo dividido por faixa etária.

Na área de Cognição pode-se notar, na Figura 7, que há maior concentração de crianças nas categorias dentro e abaixo do esperado. Porém, ainda que mais de $50 \%$ das crianças apresentem desempenho em Cognição abaixo do esperado, observa-se que no nível I a incidência é maior. É possível, no entanto, que qualquer concentração de chumbo interfira na aquisição dos comportamentos avaliados na área.

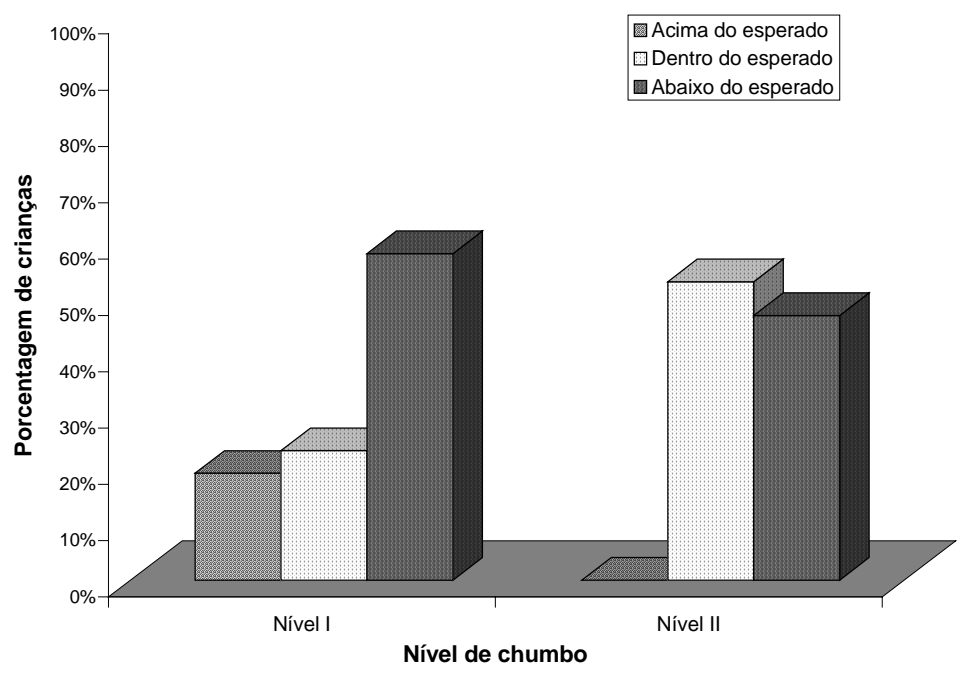

Figura 7. Desempenho em Cognição comparado ao nível de chumbo das crianças.

Ao se analisar a Figura 8, nota-se que há um alto índice de crianças com desempenho abaixo do esperado, independente do nível de chumbo. Somente na faixa etária de dois a três anos a porcentagem de crianças com desempenho abaixo do esperado é maior no nível II. Os dados obtidos podem se dever, também, ao fato de que, provavelmente, o índice de chumbo é maior nas crianças de dois a três anos pelo fato de estarem em uma fase de desenvolvimento na qual seu contato com o solo é muito grande e, tam- 
bém, na qual a criança busca experienciar o mundo através da boca, levando todo e qualquer objeto encontrado a esta de acordo com Gutiérrez (2001). Nas demais idades, o desempenho é pior para as crianças do nível I.

Segundo prova estatística de Spearman, em Cognição, somente a faixa etária de dois a três anos apre- sentou uma correlação significativa entre nível de chumbo no sangue e o desempenho obtido pela criança, confirmando os relatos de Bellinger (1995) que diz que o chumbo pode causar rebaixamento da inteligência em crianças, resultando em prejuízos acadêmicos e distúrbios psicológicos, uma vez que pode bloquear ou dificultar a transmissão nervosa.

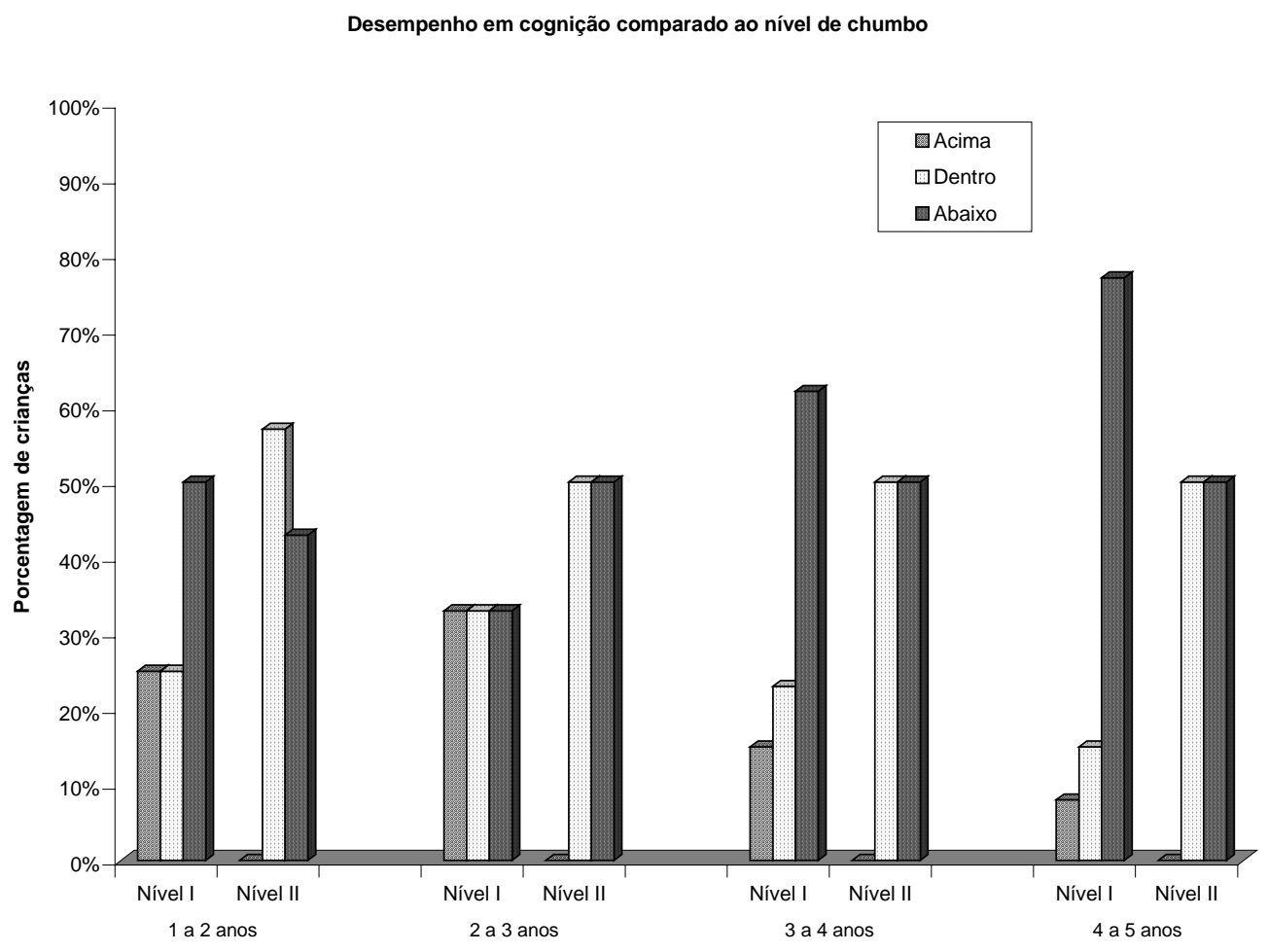

Figura 8. Desempenho em Cognição e nível de chumbo dividido por faixa etária.

\section{CONSIDERAÇÕES FINAIS}

Ainda que a literatura aponte para os efeitos do chumbo no desenvolvimento infantil, os resultados obtidos neste estudo ainda se mostraram incipientes. Nos resultados obtidos em desenvolvimento geral não se observou defasagens importantes. Considerando que o desenvolvimento geral resulta da somatória do desempenho avaliado pelas cinco áreas específicas, as mesmas foram analisadas separadamente. A análise delas apontou que as crianças, independente da faixa etária, apresentaram desempenho acima do esperado em Desenvolvimento Motor e Socialização, porém, apresentaram defasagens importantes em Cognição e Linguagem. Os dados mostraram, também, que o desempenho em Cognição piora com a idade enquanto que o de Linguagem melhora com a idade. Conside- rando o nível de chumbo temos que não há relação significativa entre os níveis de chumbo considerados e o desempenho em Cognição e Linguagem, porém, em Linguagem aparecem mais crianças em defasagem no nível mais alto do que em Cognição. Quando da análise estatística dos dados, a única área que demonstrou uma significativa relação entre nível de chumbo e desempenho foi a da Cognição, ainda para crianças na idade de dois a três anos. O número reduzido de crianças e a ausência dos dados do Grupo de Controle impedem a afirmação de que os resultados observados, ainda que coerentes com a literatura, se devam aos efeitos da contaminação por chumbo. Tais dados, entretanto, parecem indicar a necessidade de execução de grupos de controle para validá-los. 


\section{REFERÊNCIAS}

Almeida, S. H. (2003). Avaliação do desenvolvimento de crianças de um a três anos de idade contaminadas por chumbo. Monografia de especialização não-publicada, UNESP, Bauru.

Alves, C. O., Rodrigues, O. M. P. R., Figueiredo, V. A. P., Kusumi, P., \& Ribeiro, T. (2004). Desenvolvimento cognitivo de crianças contaminadas por chumbo: Resultados preliminares [Resumo]. Em Associação Brasileira de Psicoterapia e Medicina Comportamental (Org.), Anais do XIII Encontro da Associação Brasileira de Psicoterapia e Medicina Comportamental e II Congresso Internacional da Association for Behavior Analysis. Campinas: ABPMC.

Alves, C. O., Rodrigues, O. M. P. R., Troijo, M. A. F., Kusumi, P., \& Ribeiro, T. (2004). Efeito do nível de chumbo no desenvolvimento cognitivo de crianças contaminadas [Resumo]. Em Associação Brasileira de Psicoterapia e Medicina Comportamental (Org.), Anais do XIII Encontro da Associação Brasileira de Psicoterapia e Medicina Comportamental e II Congresso Internacional da Association for Behavior Analysis. Campinas: ABPMC.

Bee, H. (1986). A pessoa em desenvolvimento. São Paulo: Harbra.

Bee, H. (1997). O ciclo vital. Porto Alegre: Artes Médicas.

Bellinger, D. C. (1995). Interpreting the literature on lead and child development: The neglected role of the experimental system. Neurotoxicology and Teratology, 17(3), 201212.

Campanili, M. (2001, 14 de setembro). Resíduos industriais representam riscos para a população. O Estado de São Paulo. Retirado em 12 de agosto 2002, de http://www.estadao.com. br/ciência/noticias/2001/set/14/56.htm

Cordeiro, R., \& Lima-Filho, E. C. (1995). A inadequação dos valores dos limites de tolerância biológica para a prevenção da intoxicação profissional pelo chumbo no Brasil. Cadernos de Saúde Pública, 11(2),177-186.

Figueiredo, V. A. P., Ribeiro, M., Rodrigues, O. M. P. R., Ribeiro, T. M., \& Almeida, S. H. (2003). Efeitos da contaminação por chumbo no desenvolvimento infantil: Análise das variáveis níveis de chumbo e idade [Resumo]. Em Associação Brasileira de Psicoterapia e Medicina Comportamental (Org.), Anais do XII Encontro da Associação Brasileira de Psicoterapia e Medicina Comportamental. Londrina: ABPMC.

Gutiérrez, S. (2001). Tratamiento de la intoxicación con plomo en el niño. Boletíin Informativo de la SUP, 3(1), 11.
Malta, C. G. T., Trigo, L. A. S. C., \& Cunha, L. S. (2000). Saturnismo. Retirado em 07 de agosto de 2000, de http://www. geocities.com/HotSprings/Resort/4486/chumbo1.htm

Moreira, F. R., \& Moreira, J. C. (2004). Os efeitos do chumbo sobre o organismo humano e seu significado para a saúde. $R e$ vista Panamericana de Salud Publica, 15(2), 119-129.

Quitério, S. L., Silva, C. R. S., Arbilla, G., Moreira, M. F. R., Araújo, U. C., Mattos, R. C. O. C., \& Santos, L. S. C. (2003). Controle das emissões de chumbo particulado no entorno de uma reformadora de baterias da cidade do Rio de Janeiro usando ar como indicador. Cadernos de Saúde Pública, 19(2), 475480.

Rodrigues, O. M. P. R., Almeida, S. H., \& Ribeiro, T. M. (2003). Avaliação do desenvolvimento de crianças de um a três anos de idade contaminadas por chumbo. Em C. M. B. Neme \& O. M. P. R. Rodrigues (Orgs.), Psicologia da saúde: Perspectivas interdisciplinares (pp. 73-94). São Carlos, SP: Rima.

Silvany, N. (1996). Evolução da intoxicação por chumbo em crianças de Santo Amaro, Bahia em 1980, 1985 e 1992. Boletin de la Oficina Sanitaria Panamericana, 120, 11-22.

Stilles, K., \& Bellinger, D. C. (1993). Neuropsychological correlates of low-level lead exposure in school-age children: A prospective study. Neurotoxicology and Teratology, 15, 27-35.

Thacker, S. B., Hoffman, D. A., Smith, J., Steinberg, K., \& Zack, M. (1992). Reply: Effect of low-level body burdens of lead on the mental development of children. Archives of Environmental Health, 49, 204-5.

Wasserman, G. A. (1995). Effects of early lead exposure: Time to integrate and broaden our efforts. Neurotoxicology \& Teratology, 17, 243-244.

Williams, L. C. A., \& Aiello, A. L. R. (2001). O Inventário Portage Operacionalizado: Intervenção com famílias. São Paulo: Memnon.

Yule, W., \& Lannsdown, R. G. (1993). Blood lead concentrations in school age children, intelligence and attainment in a school population: A pilot study. Developmental Medicine and Child Neurology, 23, 567-576.

Recebido: 26/11/2006

Última revisão: 12/11/2007

Aceite final: 01/12/2007

\section{Sobre as autoras:}

Olga Maria Piazentin Rolim Rodrigues - Doutora em Psicologia Experimental, docente do Programa de Pós Graduação em Psicologia do Desenvolvimento e Aprendizagem e do Curso de Psicologia/UNESP/Bauru.

Luciana Esgalha Carnier - Licenciada em Psicologia/UNESP/Bauru e mestranda do Programa de Pós Graduação em Psicologia do Desenvolvimento e Aprendizagem, UNESP/Bauru. Bolsista PIBIC/CNPq.

Endereço para correspondência: Olga Maria Piazentin Rolim Rodrigues - Departamento de Psicologia, Faculdade de Ciências UNESP - Av. Engenheiro Edmundo C. Coube, s/nº - Bairro Vargem Limpa - Bauru/SP - CEP 17033-360

Endereço eletrônico: olgarolim@fc.unesp.br. 